Pacific Journal of Mathematics

ON EXTENDING ALMOST PERIODIC FUNCTIONS 


\title{
ON EXTENDING ALMOST PERIODIC FUNCTIONS
}

\author{
JOHN F. BERGLUND
}

\begin{abstract}
Deleeuw and Glicksberg have shown that every weakly almost periodic function on a subgroup (respectively, a dense subgroup) of a locally compact abelian topological group (respectively, an abelian topological group) extends to the whole group as a weakly almost periodic function. By way of a theorem about extending strongly almost periodic functions on subsemigroups of semitopological semigroups, we show that every almost periodic function on a subgroup (respectively, a dense subgroup) of a locally compact abelian topological group (respectively a topological group) extends to the whole group as an almost periodic function. Furthermore, for the algebra of weakly almost periodic functions on a dense subsemigroup of a semitopological semigroup to consist of restrictions of weakly almost periodic functions on the larger semigroup, we need only require that each weakly almost periodic function on the subsemigroup extend continuously. Analogous statements hold for almost periodic and strongly almost periodic functions on dense subsemigroups of semitopological semigroups.
\end{abstract}

A semitopological semigroup is a semigroup $S$ endowed with a Hausdorff topology such that the functions

$$
s \rightarrow s t: S \rightarrow S
$$

and

$$
s \rightarrow t s: S \rightarrow S
$$

are continuous. (If the function

$$
(s, t) \rightarrow s t: S \times S \rightarrow S
$$

is continuous, then $S$ is a topological semigroup.)

Let $S$ be a semitopological semigroup with identity 1. Let $C(S)$ be the $C^{*}$-algebra of bounded continuous complex valued functions on $S$. For $s \in S$, define a continuous linear operator $R_{s}: C(S) \rightarrow C(S)$ by

$$
R_{s} f(t)=f(t s), t \in S, f \in C(S) .
$$

If the closure of $R_{S} f=\left\{R_{s} f: s \in S\right\}$ in $C(S)$ is compact, then $f$ is almost periodic; if the weak closure of $R_{S} f$ in $C(S)$ is weakly compact, then $f$ is weakly almost periodic. The almost periodic functions form a sub-C*-algebra of $C(S)$, which we denote by $A(S)$. Likewise, the weakly almost periodic functions form a sub-C*-algebra of $C(S)$, 
which we denote by $W(S)$. Let $U(S)$ be the sub-C*-algebra of $C(S)$ generated by the coefficients of continuous finite dimensional unitary representations of $S$. Functions in $U(S)$ are called strongly almost periodic. For groups the strongly almost periodic and the almost periodic functions coincide.

Regarding $R_{S}=\left\{R_{s}: s \in S\right\}$ as a set of linear operators on $W(S)$ and taking its weak operator closure, we obtain a compact semitopological semigroup $\Omega S$ called the weakly almost periodic compactification of $S$. Similarly, the strong operator closure of $R_{S}$ on $A(S)$ is a compact topological semigroup $A S$ called the almost periodic compactification of $S$; and the strong operator closure of $R_{S}$ on $U(S)$ is a compact topological group $r S$ called the strongly almost periodic compactification of $S .^{1}$ Let

$$
\begin{aligned}
& \omega_{S}: S \longrightarrow \Omega S, \\
& \alpha_{S}: S \longrightarrow A S,
\end{aligned}
$$

and

$$
v_{S}: S \longrightarrow r S
$$

denote the embedding maps. There is an isomorphism of $C^{*}$-algebras $W\left(\omega_{S}\right): C(\Omega S) \rightarrow W(S)$ given by

$$
W\left(\omega_{S}\right) F=F \circ \omega_{S}, F \in C(\Omega S) .
$$

Isomorphisms $A\left(\alpha_{S}\right): C(A S) \rightarrow A(S)$ and $U\left(\nu_{S}\right): C(r S) \rightarrow U(S)$ are defined similarly. If $\varphi: S \rightarrow T$ is a morphism of semitopological semigroups, then there is a morphism $\Omega \varphi: \Omega S \rightarrow \Omega T$ of compact semitopological semigroups so that the diagram

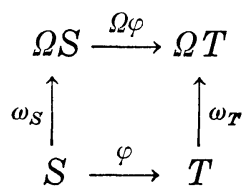

commutes. The dual diagram

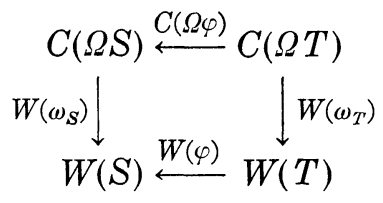

is defined naturally. There are similar morphisms $A \varphi: A S \rightarrow A T$ and $r_{\varphi}: r S \rightarrow r T$.

${ }^{1}$ One can obtain the compactifications $\Omega S, A S$, and $r S$ by way of the coadjoint existence theorem (see [1]). 
If $S$ is a subsemigroup of $T$, and if every function in a dense subset of the weakly almost periodic functions on $S$ extends as a weakly almost periodic function on $T$, then the inclusion $:: S \rightarrow T$ induces an isomorphism of semitopological semigroups between $\Omega S$ and the closure of $\omega_{T}(S)$ in $\Omega T$ (the isomorphism being the corestriction of $\left.\Omega^{\circ}\right)$. However, if $\tau \in \Omega S$, then $\tau$ is an operator on $W(S)$, whereas $\tau^{\prime}=\left(\Omega^{\prime}\right) \tau$ is an operator on $W(T)$. Nevertheless, we may identify $\Omega S$ with $\omega_{T}(S)^{-}$even as semigroups of operators. This and the analogous statements for the almost periodic and strongly almost periodic cases follow from the following lemma:

Lemma 1. Let $T$ be a semitopological semigroup with identity 1 , and let $S$ be a subsemigroup of $T$ containing 1 . Suppose that $f \in W(S)$ and that $f^{e} \in W(T)$ is an extension of $f$. Then, for every $\tau \in \Omega S$, we have that

$$
\tau f=\tau^{\prime} f^{e} \mid S
$$

where $\tau^{\prime}=\left(\Omega^{\circ}\right) \tau$.

Similar statements hold for almost periodic and strongly almost periodic functions.

Proof. We prove the weakly almost periodic case. The proofs of the other cases are completely analogous.

Consider the commutative diagram

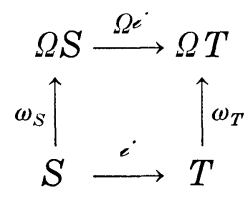

and its dual

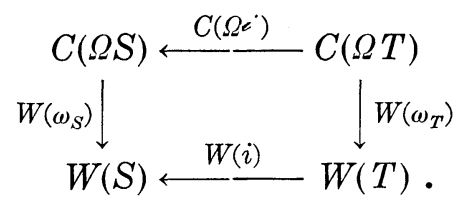

We have

$$
\begin{aligned}
\tau^{\prime} f^{e} \mid S & =W\left(\bullet^{\prime}\right) \tau^{\prime} f^{e} \\
& =\left\{W\left(\bullet^{\bullet}\right) W\left(\omega_{T}\right)\right\} W\left(\omega_{T}\right)^{-1} \tau^{\prime} f^{e} \\
& =\left\{W\left(\omega_{S}\right) C\left(\Omega \varepsilon^{\cdot}\right)\right\} W\left(\omega_{T}\right)^{-1} \tau^{\prime} f^{e} \\
& =\left\{W\left(\omega_{S}\right) C\left(\Omega^{\cdot}\right)\right\} R_{\tau^{\prime}} W\left(\omega_{T}\right)^{-1} f^{e}
\end{aligned}
$$




$$
\begin{aligned}
& =\left\{W\left(\omega_{S}\right) R_{\tau} C\left(\Omega^{*}\right)\right\} W\left(\omega_{T}\right)^{-1} f^{e} \\
& =\tau\left\{W\left(\omega_{S}\right) C\left(\Omega^{*}\right)\right\} W\left(\omega_{T}\right)^{-1} f^{e} \\
& =\tau\left\{W\left(\varepsilon^{\cdot}\right) W\left(\omega_{T}\right)\right\} W\left(\omega_{T}\right)^{-1} f^{e} \\
& =\tau W\left(e^{\bullet}\right) f^{e} \\
& =\tau f .
\end{aligned}
$$

The following lemma is immediate:

Lemma 2. Let $T$ be a semitopological semigroup, and let $S$ be a subsemigroup of $T$. Suppose that $\varphi: T \rightarrow S$ is a morphism of semitopological semigroups and a retract. Then $C\left(c^{\cdot}\right)$ maps $W(T)$ onto $W(S), A(T)$ onto $A(S)$, and $U(T)$ onto $U(S)$.

Lemma 3. Let $E$ and $F$ be Banach spaces, and let the linear transformation $\varphi: E \rightarrow F$ be an isometry. A necessary and sufficient condition that a set $K \subset E$ be weakly compact is that $\varphi K$ be weakly compact in $F$.

Proof. The necessity follows from the fact that a continuous linear transformation of Banach spaces is weakly continuous. The sufficiency follows from the fact that the adjoint transformation $\varphi^{\prime}: F^{\prime} \rightarrow E^{\prime}$ is surjective (Hahn-Banach): for if $\boldsymbol{x}$ is a net on $K$ with $\varphi \boldsymbol{x} \rightarrow \varphi x$ weakly in $F$ for some $x$ in $K$, then if $\lambda \in E^{\prime}$ and if $\theta \in F^{\prime}$ is such that $\varphi^{\prime} \theta=\lambda$, we have

$$
\begin{aligned}
\lim \langle\boldsymbol{x}, \lambda\rangle & =\lim \left\langle\boldsymbol{x}, \phi^{\prime} \theta\right\rangle \\
& =\lim \langle\varphi \boldsymbol{x}, \theta\rangle \\
& =\langle\varphi x, \theta\rangle \\
& =\left\langle x, \phi^{\prime} \theta\right\rangle \\
& =\langle x, \lambda\rangle .
\end{aligned}
$$

Proposition 4. Let $T$ be a semitopological semigroup with identity 1. Suppose that $S \subseteq T$ is a dense subsemigroup containing 1. If every weakly almost periodic function $f \in W(S)$ extends as a continuous function to $T$, then $W(S)=W(T) \mid S$.

Similarly, if $A(S) \subseteq C(T) \mid S$, then $A(S)=A(T) \mid S$; and if $U(S) \subseteq$ $C(T) \mid S$, then $U(S)=U(T) \mid S$.

Proof. For $f \in W(S)$ (resp., $f \in A(S)$; resp., $f \in U(S)$ ), let $f^{e}$ denote the (necessarily unique) continuous extension of $f$ to $T$.

(A) We show, first of all, that if $f \in W(S)$ (resp., $f \in A(S)$ ), then for each $t \in T$, there is a function $g_{t} \in\left(R_{S} f\right)^{-}$such that $R_{t}\left(f^{e}\right)=\left(g_{t}\right)^{e}$ : 
Let $s$ be a net on $S$ with $s \rightarrow t$ and $R_{s} f$ weakly convergent (resp., convergent). Let $g_{t}=\lim R_{s} f$. Then for $s \in S$, we have

$$
\begin{aligned}
{\left[R_{t}\left(f^{e}\right)\right](s) } & =f^{e}(s t) \\
& =\lim f(s s) \\
& =\lim \left[R_{s} f\right](s) \\
& =g_{t}(s) .
\end{aligned}
$$

Thus we must have $R_{t}\left(f^{e}\right)=\left(g_{t}\right)^{e}$ since $S$ is dense in $T$.

(B) Now, if $f \in W(S)$, we have that $R_{T}\left(f^{e}\right) \mid S$ is relatively weakly compact in $C(S)$; so since restriction to a dense subspace is an isometry, Lemma 3 yields that $R_{T}\left(f^{e}\right)$ is relatively weakly compact in $C(T)$.

Using the fact that restriction to a dense subspace is an isometry, we obviously have that if $f \in A(S)$, then $f^{e} \in A(T)$.

(C) Let $\mathfrak{A}(n)$ be the unitary group on $C^{n}$. Let $\xi: S \rightarrow \mathfrak{U}(n)$ be a morphism of semitopological semigroups. We show that we can extend $\xi$ to $T$ :

Suppose $t \in T$ and $s$ is a net on $S$ converging to $t$. For $x, y \in C^{n}$, we have that the function $\varphi_{x, y}: S \rightarrow C$ defined by

$$
\varphi_{x, y}(s)=\langle\xi(s) x, y\rangle
$$

can be extended as a continuous function $\varphi_{x, y}^{e}: T \rightarrow C$. Now,

$$
\begin{aligned}
\varphi_{x, y}^{e}(t) & =\lim \varphi_{x, y}(\boldsymbol{s}) \\
& =\lim \langle\xi(\boldsymbol{s}) x, y\rangle,
\end{aligned}
$$

and this must hold for every coefficient $\varphi_{x, y}$ on $\xi$. Whence, $\xi(s)$ converges in the compact group $\mathfrak{U}(n)$. Define $\xi^{e}: T \rightarrow \mathfrak{U}(n)$ by $\xi^{e}(t)=$ $\lim \xi(s)$. (This definition is independent of the particular net $s$ converging to $t$ ). By definition $\xi^{e}$ is a continuous function on $T$; since it is a morphism on a dense subsemigroup of $T, \xi^{e}$ is a morphism [1, p. 64].

(D) Now if $f \in U(S)$, then $f$ is the uniform limit of a sequence $k \rightarrow \varphi_{k}$ of coefficients of continuous finite dimensional unitary representations of $S$. By $(\mathrm{C})$, each $\varphi_{k}^{e}$ is a coefficient of a continuous finite dimensional unitary representation of $T$. It is readily seen that $f^{e}$ is the uniform limit of the sequence $k \rightarrow \varphi_{k}^{e}$.

Proposition 5. Let $S$ be a semitopological semigroup with identity 1. Let $e$ be an idempotent in the minimal ideal $M(\Omega S)$ of $\Omega S$, and let $G$ be the compact topological group e $\Omega S e$ ([1], p. 67). If $W(S)$ has a left invariant mean, then $G$ is a set of uniqueness for $\mathscr{U}=W\left(\omega_{S}\right)^{-1}(U(S))$; that is, if $F_{1}, F_{2} \in \mathscr{U}$, and if $F_{1}\left|G=F_{2}\right| G$, then $F_{1}=F_{2}$. Moreover, $F(\tau e)=F(\tau)$ for every $\tau \in \Omega S$ and every $F \in \mathscr{U}$. 
Proof. Since $W(S)$ has a left invariant mean, $M(\Omega S)$ is a minimal right ideal ([1], p. 77). Hence, $M(\Omega S)=e \Omega S$ and $\Omega S e=e \Omega S e=G$. Therefore, $\tau \rightarrow \tau e: \Omega S \rightarrow G$ is a morphism of semitopological semigroups. By Lemma 2, if $f \in U(G)=C(G)$, then $f$ may be extended to a function $F \in U(\Omega S)$ by setting $F(\tau)=F(\tau e)=f(\tau e)$ for every $\tau \in \Omega S$. From Proposition 4 one gets that $\mathscr{U}=U(\Omega S)$, so we need only observe that $R_{e} \mid \mathscr{Q}$ is injective. But that is merely the observation that $e$ is a projection of $W(S)$ onto $U(S)$ ([1], p. 83) for if $F \in \mathscr{U}$, and $f=W\left(\omega_{S}\right) F$, then $F=W\left(\omega_{S}\right)^{-1} f=W\left(\omega_{S}\right)^{-1} e f=R_{e} W\left(\omega_{S}\right)^{-1} f=R_{e} F$.

Quite simple examples of semitopological semigroups (or even topological semigroups) show that the requirement in Proposition 4 that the functions in $W(S)$ (resp., $A(S)$; resp., $U(S)$ ) extend as continuous functions to $T$ is a necessary hypothesis. However, the following example if so pathological that it may be independently interesting.

EXAMPLE 6. Let $S$ be a compact semitopological semigroup. Define the semigroup $T$ to be the set $S \times\{0,1\}$. Topologize $T$ as follows: Take neighborhoods of points $(s, 0)$ to be of the form

$$
V \times\{0\} \cup V \times\{1\} \backslash\{(s, 1)\},
$$

where $V$ is a neighborhood of $s$ in $S$; take the relative topology of $S \times\{1\}$ to be discrete. Under coordinatewise multiplication, $T$ is a compact semitopological semigroup with a dense discrete subsemigroup $S \times\{1\}$. Moreover, if $S$ has an identity 1 , then $(1,1)$ is an identity for $T$; and $M(T)=M(S) \times\{0\}$.

Now suppose that $S$ is a compact abelian group and that $\chi$ is a discontinuous character of $S$. We may consider $\chi$ to be strongly almost periodic on the diserete subsemigroup $S \times\{1\}$ of $T$; but by Proposition 5, it is clear that $\chi$ cannot be extended to all of $T$.

Lemma 7. If $H$ is a topological group, and if $G$ is a dense subgroup of $H$, then every coefficient of a continuous finite dimensional unitary representation of $G$ extends to $H$ as a coefficient of a continuous finite dimensional unitary representation of $H$.

Proof. Let $\mathfrak{M}(n)$ be the unitary group on $\boldsymbol{C}^{n}$. It is clearly sufficient to show that every morphism $\xi: G \rightarrow \mathfrak{U}(n)$ of topological groups can be extended to $H$. Observing that $\xi$ is uniformly continuous and using the fact that $\mathfrak{U}(n)$ is a complete uniform space, we get that $\xi$ extends as a continuous function. That the extension is a morphism now follows as in $4(\mathrm{C})$.

We observe in passing that the requirement that $G$ be dense is 
necessary for consider the special unitary group $G=\mathfrak{S H}(2)$ as a subgroup of the special linear group $\subseteq \&(2)$.

LEMMA 8. Let $T$ be a semitopological semigroup with identity 1. Suppose $S \subseteq T$ is a subsemigroup containing 1. Further suppose that $W(T)$ and $W(S)$ have left invariant means, and that every coefficient of a continuous finite dimensional unitary representation of $S$ extends as a coefficient of a continuous finite dimensional unitary representational of $T$. If $G$ is a maximal subgroup of $M(\Omega S)$ and $H$ is a maximal subgroup of $M(\Omega T)$, then there is a natural injection of $G$ into $H$.

Proof. Since $W(T)$ and $W(S)$ have left invariant means, the minimal ideals $M(\Omega T)$ and $M(\Omega S)$ are minimal right ideals. Let $e_{T}$ and $e_{S}$ be idempotents in $M(\Omega T)$ and $M\left(\Omega_{S}\right)$, respectively. Let $\because: S \rightarrow T$ be the inclusion map. For $\sigma \in \Omega S$, let $\sigma^{\prime}=(\Omega i) \sigma$. We want to show that the map $\sigma \rightarrow \sigma^{\prime} e_{T}: \Omega S \rightarrow H$, where $H=e_{T} \Omega T e_{T}=\Omega T e_{T}$, is injective when restricted to $G=e_{S} \Omega S e_{S}=\Omega S e_{S}$ :

Suppose that $\sigma, \tau \in G$ with $\sigma \neq \tau$. By Proposition 5, there is a function $f \in U(S)$ with

$$
\left\{W\left(\omega_{S}\right)^{-1} f\right\}(\sigma) \neq\left\{W\left(\omega_{S}\right)^{-1} f\right\}(\tau) .
$$

Since $f$ is uniformly approximated by linear combinations of coefficients $\varphi_{S}$ of continuous finite dimensional unitary representations of $S$, there must be one such $\varphi_{S}$ with

$$
\left\{W\left(\omega_{S}\right)^{-1} \varphi_{S}\right\}(\sigma) \neq\left\{W\left(\omega_{S}\right)^{-1} \varphi_{S}\right\}(\tau) .
$$

Now $\varphi_{S}$ extends to $T$ as a coefficient $\varphi_{T}$ of a continuous finite dimensional unitary representation of $T$. Since $e_{T} \varphi_{T}=\varphi_{T}$, we have that $W\left(\omega_{T}\right)^{-1} \varphi_{T}=R_{e_{T}} W\left(\omega_{T}\right)^{-1} \varphi_{T}$. So,

$$
\begin{aligned}
\left\{W\left(\omega_{T}\right)^{-1} \varphi_{T}\right\}\left(\sigma^{\prime} e_{T}\right) & =\left\{W\left(\omega_{T}\right)^{-1} \varphi_{T}\right\}\left(\sigma^{\prime}\right) \\
& =\left\{W\left(\omega_{S}\right)^{-1} \varphi_{S}\right\}(\sigma) \\
& \neq\left\{W\left(\omega_{S}\right)^{-1} \varphi_{S}\right\}(\tau) \\
& =\left\{W\left(\omega_{T}\right)^{-1} \varphi_{T}\right\}\left(\tau^{\prime}\right) \\
& =\left\{W\left(\omega_{T}\right)^{-1} \varphi_{T}\right\}\left(\tau^{\prime} e_{T}\right) .
\end{aligned}
$$

Hence, $\sigma^{\prime} e_{T} \neq \tau^{\prime} e_{T}$.

THEOREM 9. Let $T$ be a semitopological semigroup with identity 1. Suppose $S \subseteq T$ is a subsemigroup containing 1. Further suppose that $W(T)$ and $W(S)$ have left invariant means, and that every coefficient of a continuous finite dimensional unitary representation 
of $S$ extends as a coefficient of a continuous finite dimensional unitary representation of $T$. Then every strongly almost periodic function on $S$ extends as a strongly almost periodic function on $T$.

Proof. We continue with the notation of the proof of Lemma 8.

Let $f \in U(S)$, and let $F=W\left(\omega_{S}\right)^{-1} f$. By Proposition 5, $F(\sigma)=$ $F\left(\sigma e_{S}\right)$ for every $\sigma \in \Omega S$. Define $F^{\prime} \in C(\Omega T)$ as follows: Let

$$
F^{\prime}\left(\sigma^{\prime} e_{T}\right)=F^{\prime}\left(\sigma^{\prime}\right)=F(\sigma)
$$

for $\sigma \in \Omega S$, and extend $F^{\prime}$ continuously to all of $\Omega T$ by way of the Tietze Extension Theorem. That $F^{\prime}$ is well-defined follows from Lemma 8 because if $\sigma, \tau \in \Omega S$ and if $\sigma^{\prime} e_{T}=\tau^{\prime} e_{T}$, then $\sigma e_{S}=\tau e_{S}$, and we have $F(\sigma)=F(\tau)$ by Proposition 5. Let $f^{\prime}=W\left(\omega_{T}\right) F^{\prime} \in W(T)$, and let $f^{e}$ be the strongly almost periodic function $e_{T} f^{\prime} \in U(T)$. We show that $f^{e}$ is the desired extension to $T$ of the given function $f \in U(S)$ :

Let $s \in S$; then

$$
\begin{aligned}
f^{e}(s) & =\left\{R_{s} e_{T} f^{\prime}\right\}(1) \\
& =\left\{R_{s} e_{T} W\left(\omega_{T}\right) F^{\prime}\right\}(1) \\
& =\left\{W\left(\omega_{T}\right) R_{\omega_{T}(s) e_{T}} F^{\prime}\right\}(1) \\
& =\left\{R_{\omega_{T}(s) e_{T}} F^{\prime}\right\}\left(\omega_{T}(1)\right) \\
& =F^{\prime}\left(\omega_{T}(s) e_{T}\right) \\
& =F^{\prime}\left(\omega_{T}(s)\right) \\
& =F\left(\omega_{S}(s)\right) \\
& =f(s) .
\end{aligned}
$$

CoRollary 10. If $H$ is a topological group, and if $G$ is a dense: subgroup of $H$, then every almost periodic function on $G$ extends to $H$ as an almost periodic function.

Proof. By Lemma 7 every coefficient of a continuous finite dimensional unitary representation of $G$ extends as such to $H$. It is a result of Ryll-Nardzewski that the $C^{*}$-algebra of weakly almost periodic functions on a group has an invariant mean (see [1], p. 140). The result then follows immediately from Theorem 9 and the fact that almost periodic functions and strongly almost periodic functions are the same for groups.

COROLlaRY 11. Let $H$ be a locally compact abelian topological group. Suppose $G$ is a (topological, but not necessarily closed) subgroup of $H$. Then every almost periodic function on $G$ extends to $H$ as an almost periodic function. 
Proof. By Corollary, 10, we may assume that $G$ is closed. The result then follows as in the proof of Corollary 10 with the observation that characters on closed subgroups of locally compact abelian groups extend to the whole group.

\section{REFERENCES}

1. J. F. Berglund and K. H. Hofmann, Compact semitopological semigroups and weakly almost periodic functions, Lecture Notes in Mathematics 42, Springer-Verlag, Heidelberg, 1967.

2. K. de Leeuw and I. Glicksberg, The decomposition of certain group representations, J. d'Analyse Math. 15 (1965), 135-192.

Received September 9, 1969. Part of this paper represents a portion of the author's doctoral dissertation written under the direction of K. H. Hofmann at Tulane University. The author is also indebted to conversations with R. B. Burckel.

WESLEYAN UNIVERSITY 



\section{PACIFIC JOURNAL OF MATHEMATICS}

\section{EDITORS}

H. SAMelson

Stanford University

Stanford, California 94305

\section{Richard PIERCE}

University of Washington

Seattle, Washington 98105
J. DugundjI

Department of Mathematics

University of Southern California

Los Angeles, California 90007

BASIL GoRDON*

University of California

Los Angeles, California 90024

\section{ASSOCIATE EDITORS}
E. F. BECKENBACH
B. H. NeumanN
F. WOLE
K. YosHIDA

\section{SUPPORTING INSTITUTIONS}

UNIVERSITY OF BRITISH COLUMBIA

CALIFORNIA INSTITUTE OF TECHNOLOGY

UNIVERSITY OF CALIFORNIA

MONTANA STATE UNIVERSITY

UNIVERSITY OF NEVADA

NEW MEXICO STATE UNIVERSITY

OREGON STATE UNIVERSITY

UNIVERSITY OF OREGON

OSAKA UNIVERSITY

UNIVERSITY OF SOUTHERN CALIFORNIA

\author{
STANFORD UNIVERSITY \\ UNIVERSITY OF TOKYO \\ UNIVERSITY OF UTAH \\ WASHINGTON STATE UNIVERSITY \\ UNIVERSITY OF WASHINGTON \\ * * * * \\ AMERICAN MATHEMATICAL SOCIETY \\ CHEVRON RESEARCH CORPORATION \\ TRW SYSTEMS \\ NAVAL WEAPONS CENTER
}

The Supporting Institutions listed above contribute to the cost of publication of this Journal, but they are not owners or publishers and have no responsibility for its content or policies.

Mathematical papers intended for publication in the Pacific Journal of Mathematics should be in typed form or offset-reproduced, (not dittoed), double spaced with large margins. Underline Greek letters in red, German in green, and script in blue. The first paragraph or two must be capable of being used separately as a synopsis of the entire paper. The editorial "we" must not be used in the synopsis, and items of the bibliography should not be cited there unless absolutely necessary, in which case they must be identified by author and Journal, rather than by item number. Manuscripts, in duplicate if possible, may be sent to any one of the four editors. Please classify according to the scheme of Math. Rev. 36, 1539-1546. All other communications to the editors should be addressed to the managing editor, Richard Arens, University of California, Los Angeles, California, 90024.

50 reprints are provided free for each article; additional copies may be obtained at cost in multiples of 50 .

The Pacific Journal of Mathematics is published monthly. Effective with Volume 16 the price per volume (3 numbers) is $\$ 8.00$; single issues, $\$ 3.00$. Special price for current issues to individual faculty members of supporting institutions and to individual members of the American Mathematical Society: $\$ 4.00$ per volume; single issues $\$ 1.50$. Back numbers are available.

Subscriptions, orders for back numbers, and changes of address should be sent to Pacific Journal of Mathematics, 103 Highland Boulevard, Berkeley, California, 94708.

PUBLISHED BY PACIFIC JOURNAL OF MATHEMATICS, A NON-PROFIT CORPORATION

Printed at Kokusai Bunken Insatsusha (International Academic Printing Co., Ltd.), 7-17, Fujimi 2-chome, Chiyoda-ku, Tokyo, Japan.

* Acting Managing Editor. 


\section{Pacific Journal of Mathematics}

\section{Vol. 33, No. $2 \quad$ April, 1970}

Raymond Balbes and Alfred Horn, Projective distributive lattices ....... 273

John Findley Berglund, On extending almost periodic functions ......... 281

Günter Krause, Admissible modules and a characterization of reduced left

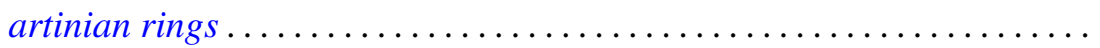

Edward Milton Landesman and Alan Cecil Lazer, Linear eigenvalues and a nonlinear boundary value problem ....................... 311

Anthony To-Ming Lau, Extremely amenable algebras ............... 329

Aldo Joram Lazar, Sections and subsets of simplexes .............. 337

Vincent Mancuso, Mesocompactness and related properties ............. 345

Edwin Leroy Marsden, Jr., The commutator and solvability in a generalized orthomodular lattice .................................. 357

Shozo Matsuura, Bergman kernel functions and the three types of canonical domains.......................................... 363

S. Mukhoti, Theorems on Cesàro summability of series .............. 385

Ngô Van Quê, Classes de Chern et théorème de Gauss-Bonnet ........... 393

Ralph Tyrrell Rockafellar, Generalized Hamiltonian equations for convex problems of Lagrange ................................ 411

Ken iti Sato, On dispersive operators in Banach lattices ............. 429

Charles Andrew Swanson, Comparison theorems for elliptic differential

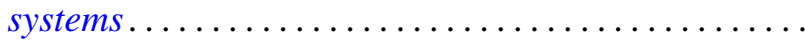

John Griggs Thompson, Nonsolvable finite groups all of whose local subgroups are solvable. II

David J. Winter, Cartan subalgebras of a Lie algebra and its ideals ... 